\title{
Self-Assembled Nanospheres: An Exciting Playground
}

\author{
G. SCHATZ \\ Department of Physics, University of Konstanz, Germany
}

Dear colleagues,

It is a difficult task to keep the balance between a whimsical after-dinner speech and a serious scientific presentation. I have decided to entertain you with, in my opinion, a very exciting aspect of nanophysics. Talking about nanostructures, there are two approaches for creation, the first one is producing these nanoobjects by lithography, the second is to copy nature and utilize self-assembly. Here I want to discuss a very simple system, namely, self-assembly of nanospheres in the diameter range between a few $\mu \mathrm{m}$ and $10 \mathrm{~nm}$ and to demonstrate with three examples what one can do with them.

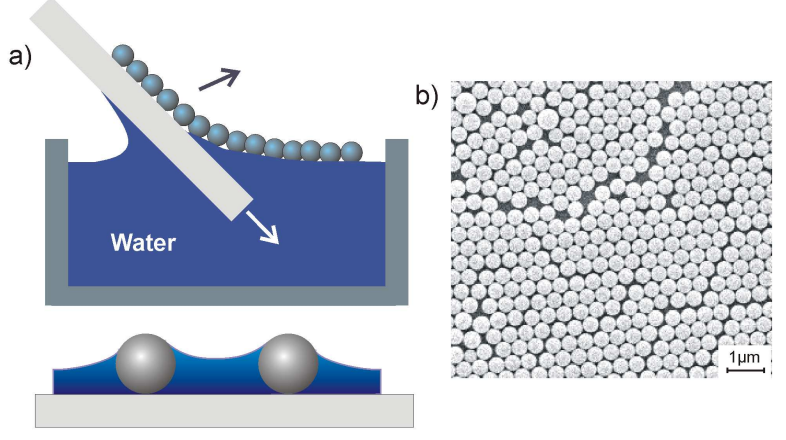

Fig. 1. Preparation process of a particle monolayer by self-assembly employing a dipping technique and capillary forces (a). SEM image of one such monolayer showing well-ordered areas as well as unavoidable imperfections (b).

Nanospheres can easily be deposited as a monolayer system by using a colloidal suspension of e.g. polystyrene nanospheres in water. A glass slide is dipped into the colloid solution and then pulled out; basically the speed determines the amount of material deposited onto the slide (Fig. 1a). The liquid then evaporates and by this exerts capillary forces, which pulls the nanospheres together and compacts them to a densely-packed monolayer. With this approach rather well-ordered areas can be achieved, but of course cracks and other imperfections can hardly be avoided (Fig. 1b).

These more or less well-ordered monolayers of nanospheres are our starting point for the production of

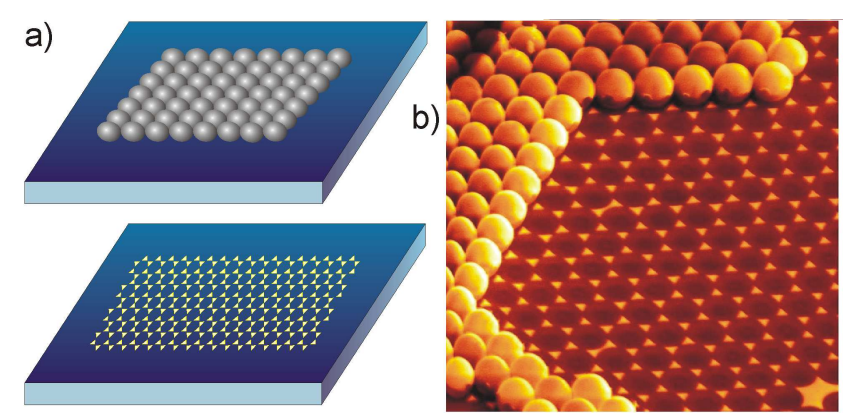

Fig. 2. In combination with film deposition, the particle monolayer can be used as template for the fabrication of two different types of nanostructures: film caps on top of the spheres and film islands between them (a). SEM image after gold deposition and partial removal of the nanospheres revealing the remaining triangular film islands on the substrate (b).

nanostructures by using them as templates for film depositions. On the one hand, the spheres are shadowing the substrate and only material can reach the substrate through the holes between the spheres [1, 2]. An image taken by electron microscopy is shown in Fig. 2b, where gold has been deposited and part of the spheres have been removed. The remaining triangular film islands form a periodic array. The side length of the triangles is in the order of the sphere radius. On the other hand, film material is covering the spheres forming caps on top of them. We will come back to these caps later, let us first see what interesting physics can be done with the triangular arrays.

The first example describes tuning of an antenna in the optical range $[3,4]$. We are focusing on just two adjacent metallic triangles, which have been produced on a silica substrate. They form a bow-tie antenna, which due to its size is expected to operate in the optical regime. The basic idea of the experiment is depicted in Fig. 3. With the tip of an atomic force microscope (AFM) one of the triangles can be shifted and the bow-tie length and the antenna gap can be tuned. The experimental setup is shown in Fig. 4 and results are depicted in Fig. 5. The experiments reveal a shift towards increasing wavelength as a function of decrease in gap size. This behavior is opposite to what one would expect from a classical metallic 
antenna, namely the shorter the antenna rod the shorter the wavelength. The observed anomalous behavior is related to the plasmon coupling between the two islands and is governed by the losses in the metal (for more details see [3]). a)

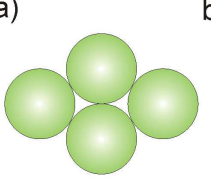

b)

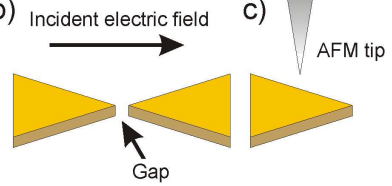

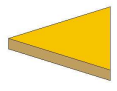

Fig. 3. Basic idea of the experiment after [4]: nanospheres act as mask for the fabrication of metallic triangles (a). The optical response to an incoming electric field is determined by the shape of the triangles and the gap size (b). An AFM tip allows shifting the triangles and therefore tuning the properties of the antenna (c).

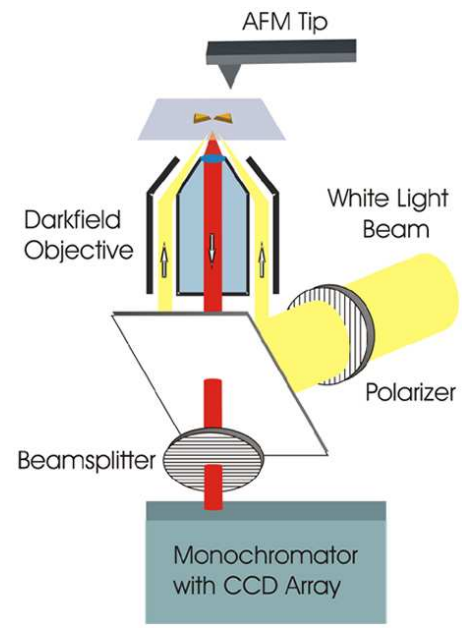

Fig. 4. The experimental setup for the investigation of the nanoantennae consists of an AFM and a dark-field scattering spectroscopy system.

In the second example laser irradiation of these triangular islands is discussed [5]. Gold triangles of about $800 \mathrm{~nm}$ side length are illuminated with a ns laser pulse. In Fig. 6 a scanning electron microscopy (SEM) image of an island region is shown where the laser intensity is increasing from the upper left to the lower right part. As one can see with increasing intensity, the islands start to melt and finally end up as molten spheres. In areas of even higher laser intensity (not shown in Fig. 6) no islands are detectable, i.e. laser ablation has occurred. In order to investigate this process the triangles have been illuminated from the back of the substrate and a catcher has been positioned at some distance from the substrate (Fig. 7). It can be clearly seen that the ablated islands have landed in a molten state. Furthermore, the

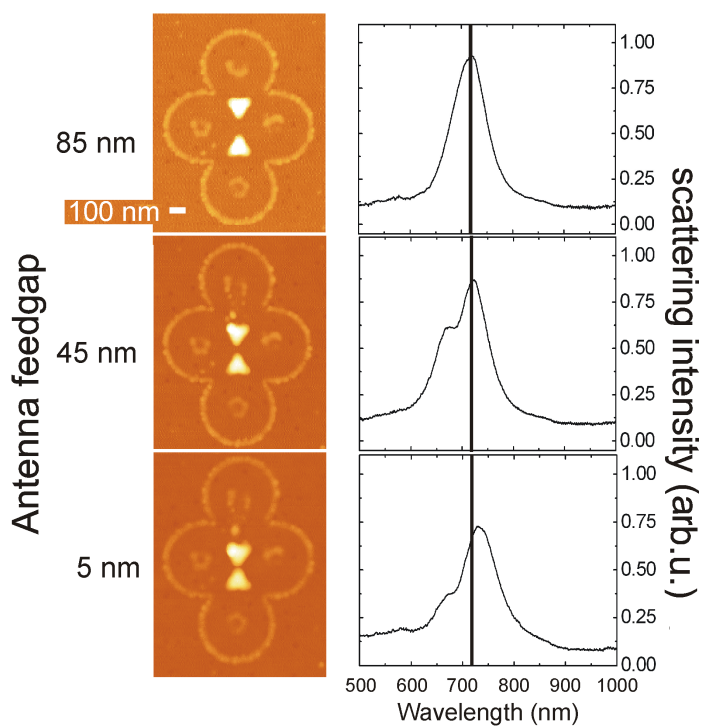

Fig. 5. AFM images (left) and dark-field scattering spectra (right) of the gold bow-tie nanoantenna for different feedgaps between 85 and $5 \mathrm{~nm}$. A shift towards increasing wavelength as a function of decrease in gap size is observed. Optical excitation and detection are polarized along the long antenna axis.

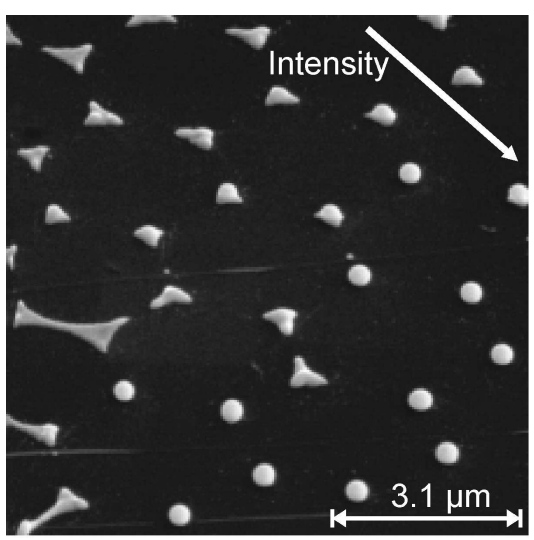

Fig. 6. SEM image of gold triangles on graphite after bombardment with a ns laser pulse. The arrow indicates the direction of increasing laser intensity. The initial side length of the triangles was $800 \mathrm{~nm}$.

velocity of the nanodroplets has been measured (Fig. 8). The traveling particles cross a second laser beam (Ar-ion laser) and the scattered light is measured with a photomultiplier. With this time-of-flight setup the velocity of the nanodroplets can be determined. Figure $8 \mathrm{~b}$ shows the result that the velocity distribution is quite narrow, considering the wide-angle emission of the nanodroplets from the substrate. The explanation of this rather narrow distribution is quite simple. During the melting process of the triangles the shape becomes more and more spherical. This releases surface energy which converts into kinetic energy of the nanodroplets. Ideal- 


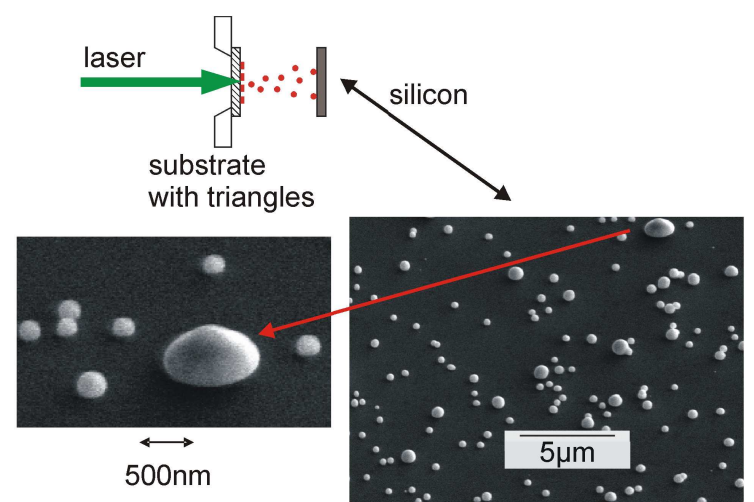

Fig. 7. For high laser intensities laser ablation occurs. The triangles are illuminated from the back side of the substrate and caught on a silicon substrate after ablation. SEM images confirm that the ablated islands have landed in a molten state.
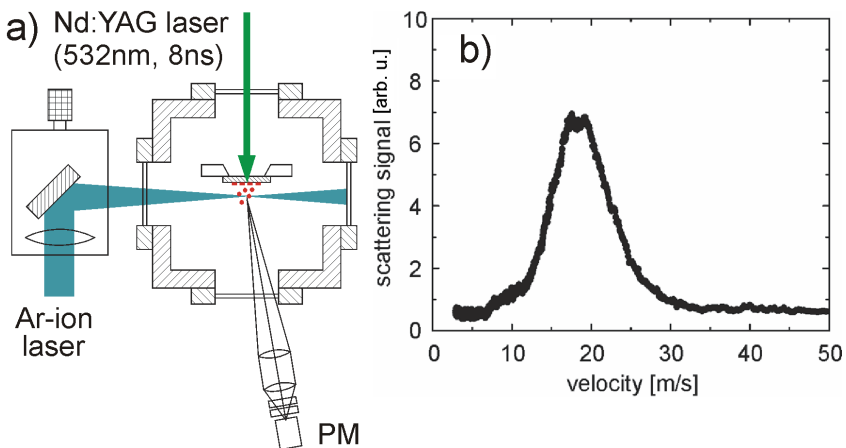

Fig. 8. Schematic drawing of the time-of-flight setup for measuring the velocity of the nanodroplets (a). For triangles with a side length of $405 \mathrm{~nm}$ a rather narrow velocity distribution is observed (b).

ized, if all triangles possess the same size and volume, the jumping nanodroplets should all have the same velocity. So far we have discussed the part of the film deposited through the spheres at the substrate and have described two interesting examples dealing with the created triangular islands. However, there is also film on top of the nanospheres forming caps (Fig. 9). Such caps have quite intriguing properties. Here an application to

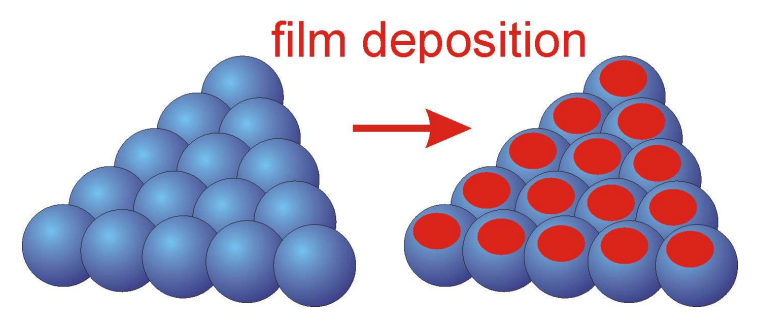

Fig. 9. Film deposition on top of a nanosphere monolayer leads to the formation of a film cap on each sphere. magnetic multilayers is presented $[6] . \mathrm{Co} / \mathrm{Pd}$ multilayers have been deposited, which exhibit a strong perpendicular magnetic anisotropy. Figure 10a shows a magnetic force microscopy (MFM) image of these magnetic caps on spheres with $270 \mathrm{~nm}$ diameter and one can see the caps either dark or bright, which clearly demonstrates a cap magnetization either up or down with respect to the substrate. A closer look also shows that the caps are magnetically decoupled, because one can find e.g. a dark cap surrounded by only bright caps. This exchange decoupling can easily be understood: the cap is thinned out towards its neighbors (Fig. 10b) and then the film loses its magnetic property. In addition to that the caps touch each other only at six very small areas, also suppressing magnetic exchange coupling.

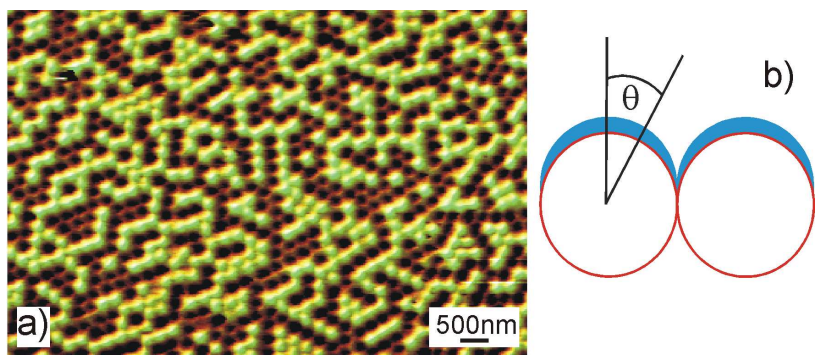

Fig. 10. MFM image of magnetic caps on spheres with a diameter of $270 \mathrm{~nm}$ after deposition of a Co/Pd multilayer film with a perpendicular magnetic anisotropy (a). Due to the curvature of the surface the film cap is thinned out from the top center towards the neighboring caps and the film loses its magnetic property (b).

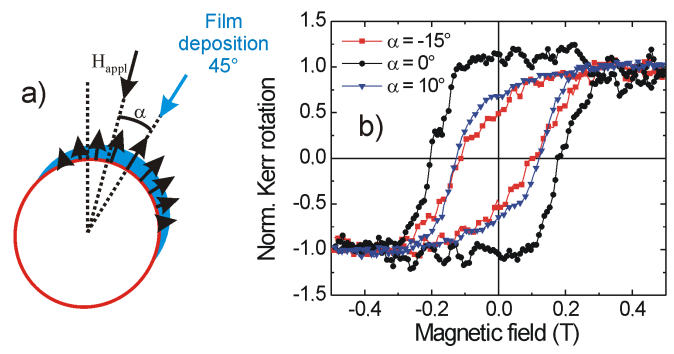

Fig. 11. Film deposition under an angle tilted with respect to the substrate plane results in a tilted average magnetization direction (a). Magnetization loops are shown for different angles (alpha) encompassing the directions of deposition and applied magnetic field. $\mathrm{Al}-$ ready for small angles $\left(\alpha=10^{\circ}\right)$ the coercitivity field is strongly reduced (about 40\%) (b). Such "tilted nanoparticle media" allow to reduce the high write fields required for high anisotropy media.

Such an array of magnetic nanostructures forms an interesting system for data recording. Since one cap would correspond to one bit, the size of the cap can be reduced down to about $3 \mathrm{~nm}$ before conflicting with the superparamagnetic limit. Moreover, the disadvantage of a very high magnetic anisotropy and consequently a strong writ- 
ing field can be lifted by depositing the multilayers under an angle tilted with respect to the substrate plane (Fig. 11). With this geometry the writing head encompasses an angle to the magnetization axes. This leads to a reduction of the coercivity field by almost a factor two [7].

In this short presentation, the variety for applications of self-assembled nanospheres as templates has been indicated and it is clear, in my opinion, that in the future many more applications will be found. Especially, the "gradient material" as represented by the caps will draw attention to the experimenters.

Finally, I want to make a very personal remark. Exactly 30 years ago I received my first invitation to attend the Zakopane School of Physics. Professor Zbigniew Bochnacki, at that time director of the Institute of $\mathrm{Nu}$ clear Physics, wrote me a letter of invitation on October 30th, 1978, asking me to attend the 17th Winter School, held in Bielsko-Biała in March 1979. This was the start of a lasting cooperation and friendship to many of my Polish colleagues.

\section{References}

[1] F. Burmeister, C. Schäfle, T. Matthes, M. Böhmisch, J. Boneberg, P. Leiderer, Langmuir 13, 2983 (1997).

[2] F. Burmeister, W. Badowsky, T. Braun, S. Wieprich, J. Boneberg, P. Leiderer, Appl. Surf. Sci. 144-145, 461 (1999).

[3] J. Merlein, M. Kahl, A. Zuschlag, A. Sell, A. Halm, J. Boneberg, P. Leiderer, A. Leitenstorfer, R. Bratschitsch, Nature Photon. 2, 230 (2008).

[4] G. Kino, Nature Photon. 2, 210 (2008).

[5] A. Habenicht, M. Olapinski, F. Burmeister, P. Leiderer, J. Boneberg, Science 309, 2043 (2005).

[6] M. Albrecht, G. Hu, I.L. Guhr, T.C. Ulbrich, J. Boneberg, P. Leiderer, G. Schatz, Nature Mater. 4, 203 (2005).

[7] G. Schatz, M. Albrecht, J. Boneberg, I.L. Guhr, US Patent, "Information storage media", 2004. 